\title{
Correction to: Metagenomics of urban sewage identifies an extensively shared antibiotic resistome in China
}

Jian-Qiang Su ${ }^{1+}$, Xin-Li An ${ }^{1,2 \dagger}$, Bing Li ${ }^{5+}$, Qing-Lin Chen ${ }^{1,2}$, Michael R. Gillings ${ }^{6}$, Hong Chen ${ }^{7}$, Tong Zhang ${ }^{4 *}$ and Yong-Guan Zhu, ${ }^{1,3^{*}}$

\section{Correction}

Unfortunately, after publication of this article [1], it was noticed that the accession numbers for the study were missing. The correct numbers are PRJNA438554, SRP148953.

\begin{abstract}
Author details
${ }^{1}$ Key Lab of Urban Environment and Health, Institute of Urban Environment, Chinese Academy of Sciences, 1799 Jimei Road, Xiamen 361021, China. ${ }^{2}$ University of Chinese Academy of Sciences, 19A Yuquan Road, Beijing 100049, China. ${ }^{3}$ State Key Laboratory of Urban and Regional Ecology, Research Center for Eco-Environmental Sciences, Chinese Academy of Sciences, Beijing 100085, China. ${ }^{4}$ Environmental Biotechnology Laboratory, The University of Hong Kong, PokfulamRoadHong Kong, China. ${ }^{5}$ Division of Energy \& Environment, Graduate School at Shenzhen, Tsinghua University, Shenzhen 518055, China. ${ }^{6}$ Department of Biological Sciences, Macquarie University, Sydney, NSW 2109, Australia. 'Department of Environmental Engineering, College of Environmental and Resource Sciences, Zhejiang University, Hangzhou 310058, China.
\end{abstract}

Received: 19 June 2018 Accepted: 19 June 2018

Published online: 09 July 2018

\section{Reference}

1. Su J-Q, An X-L, Li B, Chen Q-L, Gillings MR, Chen H, Zhu Y-G. Metagenomics

of urban sewage identifies an extensively shared antibiotic resistome in

China. Microbiome. 2017;5:84. https://doi.org/10.1186/s40168-017-0298-y.

*Correspondence: zhangt@hku.hk; ygzhu@iue.ac.cn

†Jian-Qiang Su, Xin-Li An and Bing Li contributed equally to this work.

${ }^{4}$ Environmental Biotechnology Laboratory, The University of Hong Kong, PokfulamRoadHong Kong, China

${ }^{1}$ Key Lab of Urban Environment and Health, Institute of Urban Environment,

Chinese Academy of Sciences, 1799 Jimei Road, Xiamen 361021, China

(c) The Author(s). 2018 Open Access This article is distributed under the terms of the Creative Commons Attribution 4.0 International License (http://creativecommons.org/licenses/by/4.0/), which permits unrestricted use, distribution, and reproduction in any medium, provided you give appropriate credit to the original author(s) and the source, provide a link to the Creative Commons license, and indicate if changes were made. The Creative Commons Public Domain Dedication waiver (http://creativecommons.org/publicdomain/zero/1.0/) applies to the data made available in this article, unless otherwise stated. 